\title{
Research on Remote Monitoring System of Gas Turbine Based on IoT
}

\author{
Qi Lin ${ }^{1, \mathrm{a}}, \mathrm{Li} \mathrm{Hao}{ }^{2, \mathrm{~b}}, \mathrm{Li}$ Yajing ${ }^{1, \mathrm{c}}$, Yang Yajing ${ }^{1, \mathrm{~d}}$ \\ Institute of Information Science and Engineering, Hebei University of Science and Technology, \\ Shijiazhuang, 050018 ,China \\ Pipeline Compressor-Set Maintenance Repair \& Overhaul Center of Petrol China \\ Langfang, 065000, China

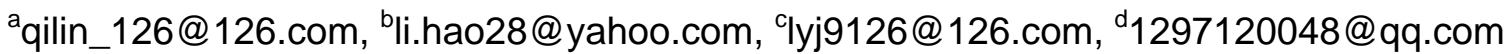

Keywords: IoT, gas turbine, real-time monitoring , platform.

\begin{abstract}
Gas turbine has been widely used in the remote transmission of natural gas. Due to its complicated structure and high temperature and high speed of bad working environment, gas turbine may break down at anytime, always lead to unplanned downtime, affect the normal capacity and have a great economic loss. At present, we lack gas turbine performance monitoring and health diagnosis on gas transmission. Thus, effective method for gas turbine healthy diagnosis is particularly important. This paper is based on the gas turbine monitoring system platform of Internet of things, constantly monitor the gas turbine performance data, effectively reduce the number of blindness of downtime and troubleshooting, which save the troubleshooting of the time, greatly improve the reliability, effectiveness and economy of gas transmission line work.
\end{abstract}

\section{Introduction}

Civil gas turbine (aviation engine is refitted machine) is used for compressor power on the west and central Asia of west-east gas pipeline, ensure the long-distance transportation of natural gas. As the heart of west to east gas pipeline, the stand or fall of gas turbine directly affect the west-east gas throughput. If for some reason the sudden failure of unplanned downtime can affect the normal delivery of natural gas ,not only bring huge economic losses, but also affect People's Daily life. At present, the gas turbine of each field is running independently, site distribution area is vast, poor working conditions ,therefore, in order to guarantee the reliability of gas turbine operation, If we build the Internet of things system, control each site through the central data center, we can real-time monitor performance parameters of gas turbine, through parameter change trend judge whether the engine work status is normal, thus early detection of potential problems in the process of operation, easy to maintenance personnel timely inspection and troubleshooting, At the same time also can better monitoring and maintenance in a planned way, and determined the work scope and depth of the gas turbine repairing, effectively allocate the spare parts of gas turbine, so as to greatly reduce the economic loss, and effectively prevent major accidents, and realize the engine maintenance on a regular basis is of great significance.

IoT is a new milestone in the development of the current information network, is widely considered to be the third wave of the world's information industry after the computer, Internet and mobile communication network. IoT technology also has been widely used in the field of intelligent agriculture, refer to division standard of the IoT system, perception layer gets all kinds of data information of cotton storehouse through the sensing technology such as the sensor, GPS, RFID and bar code, with the aid of all sorts of equipment or means; the transport layer which intergrates Wan 
and the sensor network of perception layer, transmit the information of the perception layer to the central centet fastly abd safely; processing layer completes the functions of the summary of the information, sharing, prediction and analysis and decision and so on; the application layer orients end users, set up different the operating system on the platform according to the different requirements of users.

This article apply the Internet of things technology real-time monitor the gas turbine performance parameters, and build the Gas turbine remote monitoring platform, to effectively collect the various of civil gas turbine performance parameters on the site, and analysis the diagnosis, give a warning for the problems of gas turbine. Compressor station data monitoring is mainly divided into two kinds of on-line monitoring and off-line monitoring. On-line monitoring refers to the engine in operation, by the installation of all kinds of pressure, temperature, flow rate, rotational speed, vibration sensors, such as real-time acquisition control parameters of the engine, aerodynamic parameters of the rotor rotation speed, vibration and air flow channels such as working status information, and stored in the database server. For example, the ground test, the camera record the visual image and voice of engine, and stored in the video server. Off-line monitoring refers to the engine, after stop using hole finder, fuel filter, and blockage of the lubricating oil filter and magnetic inspection, and sent data to the remote monitoring center through video or pictures.

\section{The System Architecture of IoT}

The monitoring system have two levels of local compressor station and central data center, aimed at real-time monitoring the working status of gas turbine, at the same time completing for each compressor station constitute a local monitoring site, and each local site can also transmit monitoring data to the central data center, constitute a large remote monitoring system. installing the signal detection sensor on the gas turbine of each local compressor station, connecting sensor and Zigbee module, collecting data by Zigbee node, and then transferring the data to the remote control center through the network, and using synchronization mechanism, synchronizing the local compressor station with the central information, to realize the two levels of real-time monitoring. The overall structure of the system of Gas turbine monitoring is shown in figure 1.

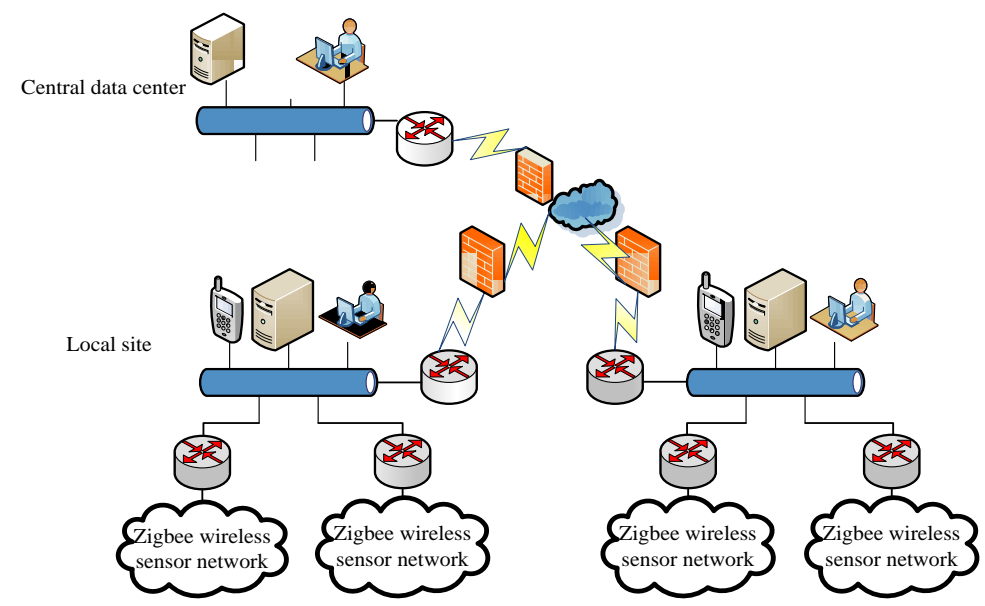

Fig 1. The Overall Structure of the System of Gas Turbine Monitoring Diagram

In order to ensure uninterrupted service of central server, the central management agencies are working with double machine hot standby mode. System adopts "Based on the Shared storage double-machine hot standby", this way is the most standard solution of the double machine hot standby. In this way two servers share the disk array storage device. Two servers can use different approaches such as case, master-slave parallel each other. In the process of work,two servers 
provide the foreign service in a virtual IP address. The server (currently tend to adopt the way of establishing private network) detects the work of another server through the heartbeat. When a server fails, another server make judgments according to the heartbeat detection conditions, and to switch and take over the service. The process is fully automatic, and the server switch can be done in a short time, don't have any impact on the business. Due to use the Shared storage devices, so two servers use the same data actually, to manage it by the dual machine or cluster software. Two foreign service server by vmware esxi 5.5 virtual made, the host server is Dell R920.

\section{The application of IoT technology in the gas turbine monitoring system}

According to the three layers architecture of the division of IoT, the remote monitoring system of gas turbine is divided into the performance of the sensing layer data acquisition, the information transmission of the network layer and application platform three parts.

Perception Layer. The sensing detection equipment of the perception layer of the Gas turbine remote monitoring system is installed on the gas turbine by gas turbine manufacturer, automatically complete the collection of data ,and sensors by using zigbee tree network layout, mainly including vibration monitoring module, the gas circuit performance module, lubricating oil monitoring module. Zigbee node module set up the network by zigbee nodes, then each module integrates into the network, finally realize the transmission of data in the network, the transmission of the data is collected by Zigbee nodes, eventually form a wireless sensor network, the communication interface of the Zigbee gathering node connect to the gateway device communication interface sends data of each each Zigbee child nodes to the remote transmission and receive the network back orders. The architecture of Zigbee wireless sensor network is shown in figure 2.

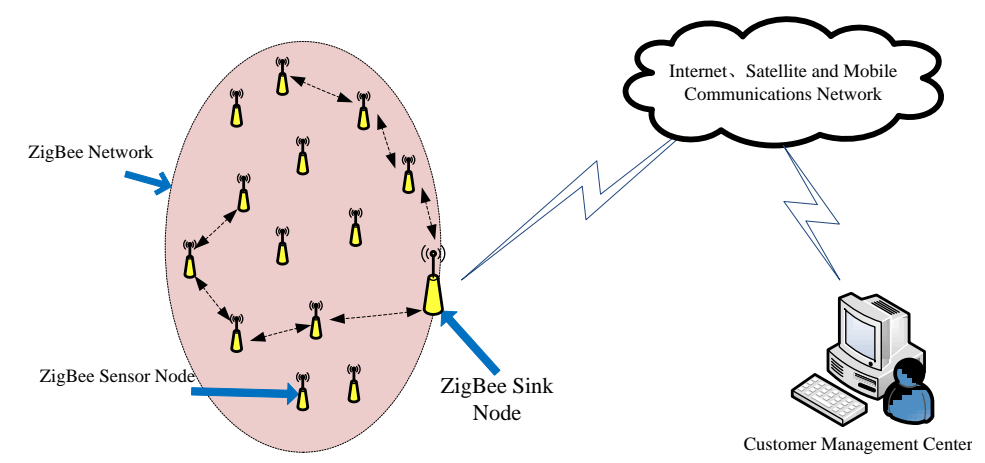

Fig 2. The Architecture of Zigbee Wireless Sensor Network

The Network Layer. The general working process to the network data transmission of the network layer of the Gas turbine remote monitoring system: send the data to the public through the perception layer Zigbee network, transimit information to the central data center through the public. due to the transparency of public is very strong, because of the strong transparency of the network, if just using the public not to take measures to protect the information, it is easy to be stolen and attacked in the transporting process, cause the leakage of national energy information. So at the transport layer use VPN technology to realize the local data transimission safely to a central data center, to ensure that the data is not being attacked and tampered by a third party during the process of transmission.

Security gateway has VPN functions, and establishes a VPN tunnel to the database server; It also has a multi-network blocking function, that can only visit the protected network by gateway after connecting the gateway client, that can block the other web page request to ensure the security of data transmission and access. The security gateway is set up at the exit of the local network in the 
cotton warehouse, that is, the internal network port of the gateway connects to the local network in cotton warehouse and the external network connects to public network, and the user is isolated from the protected network by gateway, Security gateway deployment diagram is shown in Figure 3 . The user and client initiate a request to create a tunnel and then create it, in the transmitting end of the tunnel, the user enters a password to submit a digital certificate; an encrypted connection with the client (Central data center terminal) can only be performed after the gateway authentication, the data can be transmitted after being encrypted, the data transmitted are encrypted which have already been packaged; at the central data center side, decision whether to allow the user (user or client) access or not is made according to the access control, only the user (client) that gained the access to jurisdiction and passed the authentication can decrypt the transmitted data. In this way, the maximum security of information transmission is achieved with minimum investment without changing the structure of the network. So under the premise of without changing the network structure, with minimal investment for maximum security of information transmission.

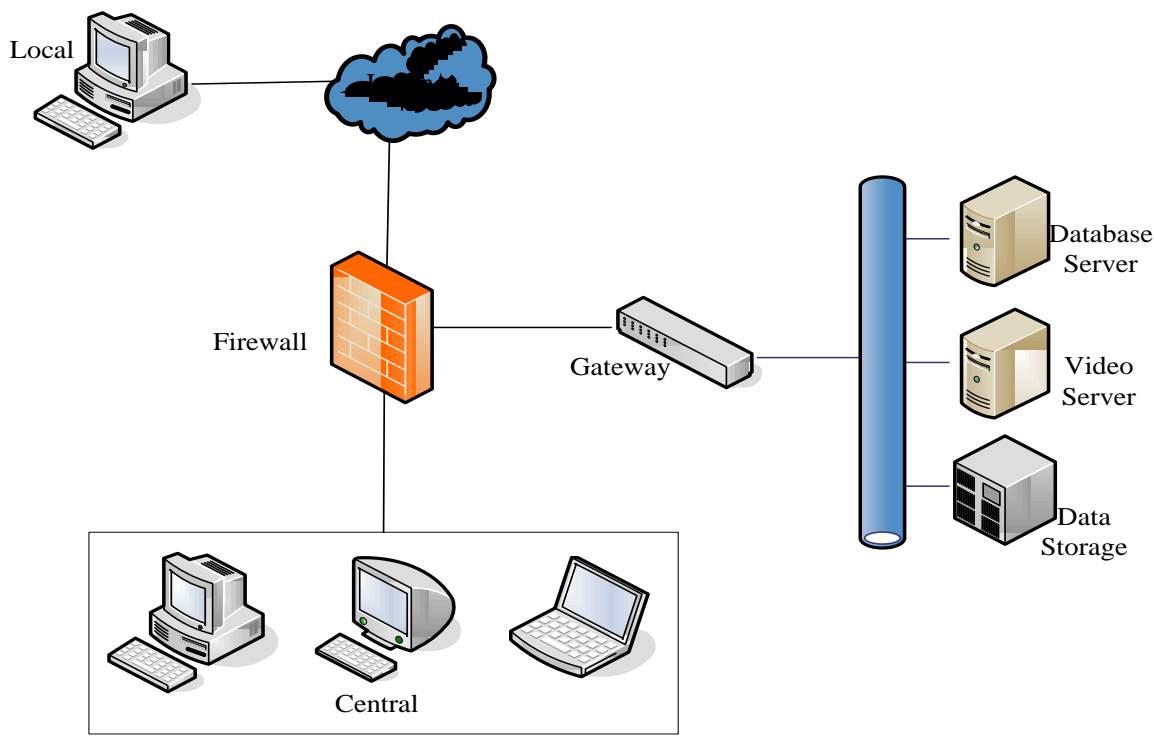

Fig.3. Security Gateway Deployment Diagram

The Application Layer. The application platform of gas turbine control system includes the performance parameters of the monitoring module, alarm and preprocessing module and management module. Mechanical vibration monitoring and diagnosis system are:

(1)The data collection: Collecting the vibration data of the unit from station monitoring system or regional headquarters compressor monitoring and diagnosis system, including real-time acquisition static data (Characteristic value of the data) and dynamic backup data on a regular basis(Vibration spectrum data).

(2)Fault early warning: According to the gas turbine structure and fault characteristics, automatic continuous records the running state of unit used the unit operation parameters such as vibration, temperature, and provide intelligent alarm respectively to the amplitude, fault characteristics and the trends.

(3)Vibration analysis: Use a variety of mapping tool to the analysis of fault diagnosis. Including: centrifugal compressor map: time domain analysis, frequency spectrum analysis, phase analysis, the axis trajectory, axis location map, trend graph, bar chart and single value stick figure, polar diagram, holographic spectrum, full spectrum, Nyquist diagrams, $3 \mathrm{~d}$ waterfall spectrum diagram, potter, cascade figure.

(4)Reciprocating compressor map: The analysis diagram of the operation of the unit state, vibration monitoring diagram(can realize the trend of the measuring point, waveform and 
spectrum analysis, and basic information display), impact monitoring figure, piston rod position monitoring diagram, history comparison chart, single value stick figure, multi-parameter analysis, comprehensive monitoring, the piston rod path chart.

(5)The function of releasing the alarm information: Using the short message of mobile phone and emails.

The module of Gas circuit performance:

(1)The data collection: From the UCP contains collect The unit state parameter.

(2)the status display unit: Real-time (refresh rate is $1 \mathrm{~s}$ ) display the state parameters of the unit.

(3)the fault alarm: Real-time display the fault alarm information of the unit.

(4)Parameter trends show: Mainly including: the exhaust temperature, inlet temperature, oil pressure, oil temperature, speed, power, efficiency, decay, load rate and other important parameters.

(5)The performance analysis of gas turbine : the figure shows of the efficiency of the Compressor working condition, gas turbine power and efficiency calculation, flow calculation, etc.

(6)The life prediction of the gas generator and key to life prediction.

The data monitored by the Performance parameters including: 1)Gas circuit and performance trends. Mainly including gas turbine inlet temperature, pressure and humidity; Medium voltage compressor outlet temperature and pressure; High pressure compressor outlet temperature and pressure; The turbine exhaust temperature and pressure. 2)Lubricating oil related data. Mainly including the lube oil supply oil pressure, oil pressure; Lubricating oil temperature; Lubricating oil debris monitoring (MCD). 3)The related information of bearing vibration. Mainly including gas turbine front support bearing vibration parameters; In support of bearing vibration parameters; After the support bearing vibration parameters. 4)Speed. Mainly including low speed axis; High speed axis; Start the machine speed. 5)The adjustable guide vane position (VIGV). 6)the life of the lifetime. The life of the life is mainly to monitoring the life of the core components in the gas turbine, such as high finale life.

Alarm and preprocessing module is mainly to manage the performance parameters of gas turbine, used to identify the abnormal data by extracting the data in the database and according the result of recognition to generate the analysis file; It also can send out alarm information immediately for the data beyond the normal limits and the Coming to the life of the parts the machine automatically stop or artificial down to prevent damage. According to the gas turbine performance trend of gas path, lube oil data, velocity data, the vibration data of hole drilling information, when the report information such as the life of a lifetime and the unit, the engineer of the Central data center can comprehensibly judge the cause of the problem, find out the fault location, formulate solutions, and feedback through the remote monitoring platform to local staff, guidance on site to repair or replace new parts.

The management module is mainly to mange the performance of the gas turbine, establish the resume of gas turbine information, to statistics all the engine parts using information For each air site, to change the coming to the life of the parts, to distribute in time and repairing to be gas turbine of coming to the life; To order the spare parts and gas turbine On demand Based on the analysis of the data in the database, ensure overall deployment, implementation "lean manufacturing" and "zero inventory" storage, change the traditional thought of putting prevention in the blindness, according to the regulations of the OEM quantitative orders regularly we lack of spare parts, not only guarantee the safety in production, but also save the cost effectively, improve the fine management.

\section{Conclusion}

According to the three layer architecture of the Internet of things, we design the system of gas turbine monitoring,improve the efficiency of the fault diagnosis and troubleshooting by contacting 
the monitoring of the scene, troubleshooting and diagnosis expert, realize the reliability of gas turbine running and maintenance mode from the traditional periodical maintenance to according the change of situation,make up for the traditional cannot according to the gas turbine performance data analysis of potential failure. This system can also Order the spare parts based on the database data in the platform of the Internet of things ,not only guarantee the safety in production, but also save the cost effectively, improve the fine management. This system can also improve the efficiency and quality of office in combination with the office automation system.

\section{References}

[1] Chen Minghai, Cui Li,Xie Kaibin. A Comparative Study on Architectures and Implementation Methodologies of Internet of Things,2013,01:168-188.

[2] Li Zeping. Research and design of improved business activity real-time monitoring platform[J] Computer application research, 2012,03:988-990+997.

[3] Qian Zhihong, Wang Yijun. The Internet of things technology and applied research[J]. Electronic journals, 2012,05:1023-1029.

[4] Cai Jiran, Cao Haichuan. Real-time monitoring system based on OPC and Comet with B/S architecture[J]. Computer application, 2012,S2:214-216.

[5] Ai Yong. Key Technologies Research for Monitoring Platform Based on Nagios [D]. University of electronic science and technology, 2013.

[6] Lu Jialong. Design and Implemenation of Real-Time Monitoring System Based on Virtual Instrument [D]. Harbin institute of technology,2013.

[7] $\mathrm{Yu}$ Cunshui. Design and Implementation of Monitoring Platform Based on Smart Grid Scheduling-System[D]. Jilin university,2013.

[8] Cheng Yuan. Research on maintenance interval optimization strategy model of gas turbine passage parts[D]. Zhejiang university,2014.

[9] Gong Zheng. Network Video Monitoring Platform Software Research and Implementation[D]. Dalian university of technology,2012.

[10]Zhang Xiaodong. Investigation on Optimization Design Method of Gas Turbine Aerodynamic and Cooling [D].Graduate school of Chinese academy of sciences(Institute of engineering thermal physics),2012.

[11]Sun Lanxin. Study on Wet Compression Performance of Gas Turbine and Droplet Motion [D]. Harbin engineering university,2012.

[12]Huang Yi. Research on Reconfigurable Manufacturing Execution System for RFID-based Real-Time Monitoring [D]. Tsinghua university,2011. 